\title{
Casal Jove Atlas Un caso de co-diseño para la intervención social ${ }^{1}$
}

\section{Resumen}

Desde finales de los años noventa, el diseño ha ampliado su radio de acción. Ahora los diseñadores, también, emplean sus conocimientos, metodología y habilidades en proyectos sociales, con la finalidad de empoderar a las personas, impulsar su participación y su vinculación a la comunidad, así como para evaluar situaciones que precisan de una intervención social. En este artículo se presenta brevemente un proyecto de diseño para la intervención social dirigido a jóvenes en riesgo de exclusión del barrio del Raval de Barcelona.

Palabras clave: Diseño social, Trabajo Social, intervención social, jóvenes en riesgo de exclusión.

\begin{abstract}
Since late 1990s, design has broadened its scope. Now designers also use their knowledge, methodology and skills in social projects, with the aim of empowering people, encourage their participation and their link within the community, as well as to assess situations that require a social intervention. This article briefly presents a design project for social intervention aimed to young people at risk of social exclusion in the Raval neighborhood of Barcelona.
\end{abstract}

Keywords: Social design, Social Work, social intervention, young people at risk of exclusion.

Agradezco la inestimable y desinteresada colaboración de todas las personas que han intervenido en este proyecto: Martin Dubuisson, Aurélie Glorieux, Mercè Graell, Alessandra Luis (equipo de Designit); Alicia Gómez y Pilar Villuendas (estudio Villuendas + Gómez); Jordi Navarro y Maria Vinuesa (Associació de Mestres Rosa Sensat); Anna Guiot, Pep Quílez y Clara Salvador (Secretaría de ADG-FAD); Sara Ruiz y Josep Vives (Casal Jove Atlas). Gracias también a los jóvenes que participaron, cuyos nombres no menciono por motivos de confidencialidad. 
El propósito de los diseñadores de contribuir a la transformación social del entorno se ha visto acelerado desde 2008, coincidiendo con el incremento de la desigualdad social y la pobreza, los desastres medioambientales, la inestabilidad política y los conflictos bélicos que, en estos momentos, asolan al planeta.

El diseño está ampliando su radio de acción y ahora, además de seguir proyectando artefactos, espacios y comunicaciones, los diseñadores emplean su metodología de trabajo en proyectos de intervención social ${ }^{2}$. De este modo, se está produciendo un acercamiento al Trabajo y la Educación Social, lo que, desde mi punto de vista, convierte al diseño en una profesión cada vez más relacional, en la que el cuidado del otro, la ayuda, la capacidad de comunicación de los profesionales y la relación interpersonal son fundamentales.

Victor y Sylvia Margolin (2002) ya vislumbraban ese acercamiento al esbozar un modelo de diseño socialmente responsable basado «en el modelo de intervención utilizado por los trabajadores sociales» (p.25), para aplicarlo en proyectos de salud y enseñanza, en colaboración con otros profesionales de la asistencia. Proponían, además, basarse en las perspectivas ecológica y sistémica ${ }^{3}$, empleadas por los trabajadores sociales, y en la metodología que estos utilizan para la resolución de problemas.

Desde que se publicó el artículo de Margolin y Margolin, las prácticas colaborativas se han incrementado; los diseñadores están empleando métodos que tienen puntos en común con los de los trabajadores sociales y están alineados, (incluso sin ser conscientes de ello) con las perspectivas sistémica y ecológica ya mencionadas. Esto permite pensar que el diseño socialmente responsable se está acercando al modelo social propuesto por estos autores.

Precisamente, la necesidad de indagar en ese modelo me ha llevado, desde el año 2014, a investigar en la relación entre diseño y Trabajo Social ${ }^{4}$ y a trabajar con colectivos en dificultad social, en un intento de explorar de qué manera puede el diseño contribuir a cambiar su situación y cuáles son sus límites y posibilidades. En este artículo presento brevemente una de las experiencias que he llevado a cabo en el Casal Jove Atlas de Barcelona.

2 Entendemos por intervención social: «una acción programada sobre un colectivo o grupo con el fin de provocar un cambio social para mejorar su situación. La intervención consiste en una serie de actividades y tareas programadas con detalle y con una metodología de trabajo concreta destinadas a la consecución de un fin» (Sandra Losada, 2016, p. 16).

3 La perspectiva sistémica se relaciona con la aplicación de la teoría de sistemas del biólogo Ludwig von Bertanlanffy (1976). En el Trabajo Social aporta una visión holística de las relaciones interpersonales y proporciona la posibilidad «de contemplar a la persona/ situación como un todo de elementos relacionados entre sí» (Segado Sánchez-Cabezudo, 2011, p. 41). En la perspectiva ecológica se defiende que la dimensión ambiental tiene un papel fundamental en las condiciones de vida de los seres humanos y que para entenderlos es preciso tener en cuenta el contexto de relaciones que se dan entre ellos. En el Trabajo Social, se centra en descubrir las fortalezas y debilidades de los individuos y en los procesos transaccionales entre las personas, familias, culturas o comunidades (Segado Sánchez-Cabezudo, 2011).

4 Para profundizar en mis investigaciones, he estudiado un Máster Universitario en Trabajo Social, Estado del Bienestar y Metodologías de intervención social. 


\section{El Casal Jove Atlas}

Situado en el barrio del Raval ${ }^{5}$ de Barcelona, el Casal Jove Atlas es un centro para jóvenes, dependiente del Casal dels Infants ${ }^{6}$. Espacio de ocio y de participación comunitaria, acoge a jóvenes, de entre 15 y 21 años, que se encuentran en riesgo de exclusión social ${ }^{7}$ debido a las situaciones socioeconómicas en las que viven o por su condición personal y/o de inmigrantes.

Las actividades que ofrece son fundamentalmente deportivas, de ocio y de ayuda al estudio. Con ellas se pretende fomentar «el desarrollo de competencias, reforzar la red social de apoyo y favorecer el itinerario de inserción de cada joven» (Ruiz, 2016), a través de los siguientes contextos:

- Asambleas, un espacio comunitario y democrático para trabajar la escucha, la asertividad, la empatía y el sentimiento de pertenencia a la comunidad.

- Grupos de trabajo por proyectos concretos. Por ejemplo, comisión de actividades comunitarias, de organización de salidas, etc.

- Espacios de intervención grupal, con actividades de ocio relacionadas con el deporte o el arte y el espacio de encuentro.

- Espacios de intervención comunitaria, de relación con el territorio y las personas que conviven en él.

- Espacios de intervención individual: las entrevistas y el área de estudio. Facilitan el tratamiento de situaciones particulares y el seguimiento de los planes de trabajo individualizados.

Desde una perspectiva de atención sistémica y holística, en el Casal se detectan situaciones de vulnerabilidad; se acompaña a los jóvenes en su evolución personal de manera individualizada y se les ayuda a aumentar las competencias necesarias para la toma de decisiones; se les conecta con los recursos del territorio que puedan cubrir sus necesidades y con la cultura local; se les proporciona un tratamiento multidimensional dirigido a estimular sus potencialidades y se les ofrece, como ya he comentado más arriba, soporte para que puedan finalizar con éxito su itinerario académico.

\section{Los jóvenes del Casal}

En el contexto de la sociedad del nuevo milenio, profundamente marcada por la globalización, las transiciones a la vida adulta de un buen número de jóvenes son cada vez más tardías y complejas, propiciando un modelo que se ha denominado de «jóvenes adultos»: «jóvenes que aún no han transitado de forma clara a una vida

5 En 2017, el Raval contaba con una población de 47.986 vecinos empadronados. De estos, 23.818 habían nacido en el extranjero. El vecindario estaba formado por 122 nacionalidades. En cuanto al número de jóvenes, de entre 15 y 24 años, en el barrio vivían 4.890 . Si bien el índice de renta familiar disponible per cápita no es el más bajo de la Barcelona, en el barrio existen zonas de pobreza extrema y de exclusión severa.

6 El Casal dels Infants es una asociación para la acción social en los barrios, fundada en 1983.

7 Siguiendo la definición de Melendro et al. (2014), se entiende por riesgo de exclusión: «una situación de desventaja, dificultad o imposibilidad en el acceso a los mecanismos de desarrollo personal, de inserción comunitaria y a los sistemas de protección social, así como a una privación de la propia idea de ciudadanía, de los derechos y libertades básicas de las personas» (s.p.). 
adulta autónoma, independiente. Alcanzan los diferentes niveles de independencia -laboral, social, familiar, económica- cada vez a una edad más tardía y de forma menos lineal, más sinuosa y aleatoria» (Melendro, 2009, p. 25).

Pero si esta es una situación que, actualmente, afecta a la mayoría de los jóvenes, los que se encuentran en situación de dificultad social son especialmente vulnerables. Entre las características personales que estos comparten están la baja autoestima, «la dificultad para anticipar las consecuencias de sus actos y verbalizar sus sentimientos, la impulsividad unida a componentes agresivos, la escasa tolerancia a la frustración, una necesidad permanente de estimulación, es decir, de encontrar espacios y situaciones en los que hallar niveles de satisfacción satisfactorios y, junto a todo ello, importantes dificultades de atención, concentración, memoria, expresión, comunicación y relación» (Melendro, 2009, p. 62). Estos rasgos, unidos a trayectorias familiares complejas y a situaciones económicas de precariedad, propician que tengan menos oportunidades en el futuro y más posibilidades de verse afectados por la pobreza y la exclusión social.

Centrándonos en el perfil de los «usuarios» del Casal:

- Principalmente son inmigrantes o de otras etnias.

- Proceden de familias con bajos (o muy bajos) niveles económicos y culturales. En algunos casos, forman parte de familias desestructuradas con patrones de relación inadecuados.

- Algunos de ellos pertenecen a familias usuarias de servicios sociales.

- Parte de ellos tiene un historial de fracaso escolar que incide negativamente en su motivación y autoestima.

- Experimentan dificultades para enfrentarse a las relaciones interpersonales, a la búsqueda de empleo y al aprendizaje.

- Poseen baja resistencia a la frustración y poco control de las situaciones. Tienen poca motivación de logro.

- Son usuarios habituales del Casal Jove y algunos de ellos lo han sido de otros centros del Casal dels Infants (Casal Obert Infantil y Casal Obert Adolescent) y lo son todavía (Centre de Formació i Inserció).

- Se hallan en riesgo de exclusión social.

A esto hay que añadir que se encuentran con modelos escolares rígidos y paternalistas que mantienen unos curricula monoculturales (Martínez-Celorrio, 2015). Por eso necesitan una atención personalizada y respaldo a la hora de resolver los conflictos que son propios de su momento evolutivo y, también, que se les valore para contrarrestar su autodesvalorización, debida a las situaciones y contextos en los que se desenvuelven sus recorridos vitales. No obstante, como la mayoría de los jóvenes:

- son más creativos de lo que ellos mismos piensan y tienen curiosidad natural.

- Son capaces de cuestionar lo establecido y de buscar alternativas.

- Están abiertos a nuevas experiencias y a aprender cosas nuevas.

- Si se sienten motivados, son capaces de implicarse en lo que se les proponga. 


\section{Diseño para la intervención social en el Casal Jove Atlas}

Mis primeras intervenciones en el Casal se efectuaron con los grupos de trabajo por proyectos (las "comisiones»), fundamentados en la idea de que los jóvenes reciben ayuda del Casal pero, a cambio, tienen que devolver algo a la comunidad, en forma de una dedicación más bien simbólica de su tiempo (una hora a la semana), haciendo propuestas que mejoren el centro o el barrio. Con ello se intenta romper con el asistencialismo, transmitiéndoles que son miembros de una sociedad a cuyo bienestar han de contribuir.

Las «comisiones» se insertan dentro de un proyecto socioeducativo amplio y se han orientado, precisamente, a cumplir los propósitos de promover la autonomía y el crecimiento personal de los jóvenes. A la hora de configurar las actividades en las que intervienen las herramientas del diseño, se ha tenido en cuenta que, entre las dificultades con las que tropiezan cotidianamente, está la de afrontar los problemas y tomar decisiones. Por ello, se ha considerado esencial estimular su creatividad, con el fin de que puedan «convertir los problemas en oportunidades» (De Prado como se citó en Del Val, 2011, p. 18).

Para activar los procesos creativos se han empleado herramientas de ideación como, por ejemplo, fichas de definición de ideas, el collage, el brainstorming, SCAMPER, el brainwrite y la Worst Possible Idea, entre otras, así como de identificación de problemas (por ejemplo, fichas de definición del reto y refinado de soluciones), los mapas de empatía y el método personas (para describir al usuario y su mundo).

De las comisiones han surgido ideas como, por ejemplo, la de realizar diversas acciones que mejoraran las relaciones con la policía dentro del barrio, una fiesta para recaudar fondos y donarlos a las personas «sin techo", crear un pequeño huerto urbano o mejorar el interiorismo del Casal, para hacer de él un espacio más intercultural pues, aunque allí confluyen jóvenes de diferentes culturas, no siempre la comunicación entre ellos es fluida. Por diversas circunstancias8, esas propuestas no han podido hacerse realidad, aunque la segunda dio lugar a un taller (junio de 2017) en el que los jóvenes construyeron mobiliario con palés, junto a estudiantes del Grado de Diseño de la Universidad de Barcelona y de la Escuela Massana, también de Barcelona. Dicho taller nos permitió centrarnos en aspectos relacionados con el trabajo en grupo y sirvió de acercamiento entre jóvenes pertenecientes a contextos sociales bastante diferentes. Por otra parte, las herramientas del diseño aplicadas a las «comisiones» han resultado útiles para generar dinámicas de grupo y para profundizar en el conocimiento de las características personales de los participantes.

Cabe recordar que los proyectos artísticos y de diseño aplicados a la intervención social han puesto de relieve que, basándose en la creatividad, se fomentan: «las relaciones entre las personas y contribuyen a construir marcos de interacción que revierten en los participantes y la comunidad en la que viven» (Ricart y Saurí, 2009, pp. 14-15). Ricart y Saurí (2009) comentan que los proyectos artísticos aportan a los participantes en el proceso visibilidad y reconocimiento, oportunidades porque contribuyen a construir puentes entre las personas y las instituciones, a establecer relaciones e interacciones, -al crear redes entre los participantes-, producen nuevas formas de comprender y de relacionarse con el entorno, generan emociones positivas y un proceso de empoderamiento que impulsa una transformación tanto personal como social.

Sin ser un proyecto artístico, el trabajo con los jóvenes del Casal se inserta en esta línea puesto que el diseño es una disciplina creativa que se mueve entre la estética y el arte, pero también entre la ciencia, la técnica y la tecnología.

8 Entre esas circunstancias, está que muchos de estos jóvenes no asisten con regularidad al Casal porque tienen obligaciones familiares, se incorporan a puestos de trabajo temporales, etc. 
Ahora bien, aunque la creatividad ha tenido un papel fundamental en las acciones realizadas en el Casal, ha sido un medio más dentro de un proceso en el que también se mezclan otros componentes de igual importancia como son la participación, el empoderamiento, la relación y la interacción, la visibilidad, el reconocimiento y el diálogo, entre otros. La aspiración ha sido siempre la de contribuir a reforzar las capacidades de los jóvenes y su autoestima; en el plano colectivo, se ha pretendido mejorar sus habilidades de comunicación y de trabajo en equipo.

Además de la experiencia de las «comisiones», en abril de 2017 hicimos un pequeño proyecto consistente en el co-diseño del cartel anunciador de un evento organizado por una asociación de maestros de Barcelona.

\section{Co-diseño y Design thinking}

Aunque todavía no existe un consenso sobre la definición del término co-diseño, este sirve para designar una metodología basada en el trabajo con los usuarios finales de un producto o servicio para desarrollarlos de una manera más eficaz. Sanders y Stappers (2008) indican que es la creatividad colectiva aplicada a un proceso de diseño. Se considera una metodología democrática, basada en la colaboración y la participación entre los clientes/usuarios y los diseñadores, todos ellos situados en un plano de igualdad. De ahí que se perciba como especialmente adecuada para abordar problemas sociales complejos y para aportar soluciones innovadoras, en la medida en que se emplean técnicas que implican a las personas que conocen y comprenden esos problemas de una manera más completa y profunda.

Entre los métodos de co-diseño se encuentra el Design thinking. Las primeras referencias a él se remontan a la década de 1960. Por aquel momento, el término se relacionaba con cómo pensaban y actuaban los diseñadores dentro del proceso de diseño y, sobre todo, con cómo resolvían problemas. A partir de los años noventa fue cuando comenzó a considerarse que esa manera de pensar podía ser útil en otros campos externos al diseño. Actualmente se ha convertido en una herramienta para aquellas empresas y organizaciones que intentan potenciar la innovación mediante la creatividad. El método ha demostrado ser especialmente eficiente a la hora de identificar problemas. En ese sentido, las organizaciones sin ánimo de lucro han comenzado a ser conscientes de sus ventajas para conseguir mejores soluciones a los problemas sociales con los que han de enfrentarse. De ahí que se esté hablando del Design thinking para la innovación social.

\section{El taller}

En noviembre de 2016, la Asociación de Maestros Rosa Sensat ${ }^{9}$ de Barcelona, se puso en contacto con la Asociación de Diseñadores Gráficos y Directores de Arte del FAD (ADG-FAD), para explorar la posibilidad de que algún diseñador se encargara de crear un cartel para anunciar su Escuela de Verano. Tras mantener una entrevista con ellos, y dada la naturaleza social de la entidad, se les propuso una alternativa: que ese cartel surgiera de un proyecto de intervención socioeducativa, llevado a cabo con los jóvenes del Casal Jove Atlas.

El proyecto consistió en un taller de co-diseño, aplicando el Design thinking como método. Se inició con un

9 La Asociación de Maestros Rosa Sensat se constituyó en Barcelona en 1982 pero sus orígenes se remontan a 1965, cuando surgió como movimiento de renovación pedagógica con la finalidad de mejorar la educación. Entre los ámbitos en los que se desarrollan las actividades de la organización, está el de la formación permanente, a través de cursos, seminarios y grupos de trabajo y la escuela de verano, que se organiza desde 1966. En este proyecto se contó con la implicación de Maria Vinuesa Arbós (Comisión Ejecutiva) y Jordi Navarro (Jefe de Comunicación y Prensa) 
encuentro con los representantes de la Asociación para que los jóvenes conocieran la finalidad del proyecto. Con esto se perseguía que se acercaran a otras realidades distintas a la suya y, más concretamente, a la de la educación vista desde la perspectiva de los maestros.

Aunque no hubo un proceso de selección por parte del equipo del Casal, _porque se consideró más conveniente la participación voluntaria-, se inscribieron doce jóvenes. De manera natural se consiguió un equilibro numérico entre chicos y chicas, importante en la medida en que en los proyectos del centro se contempla la perspectiva de género. También estuvo presente el enfoque intercultural porque es una realidad del barrio.

Tras una semana para que los interesados confirmaran su colaboración en el proyecto, se realizó un taller de doce horas, repartidas en cuatro sesiones: 9 horas de co-diseño, distribuidas en tres días y guiadas por un equipo de diseñadores especializados en diseño de servicios, más otras 3 horas de trabajo con una diseñadora gráfica.

Entre las herramientas empleadas por los diseñadores encargados del taller de co-diseño pueden mencionarse el collage, los mapas de empatía, la construcción de prototipos con Lego y el dibujo rápido. En este taller se recopiló y analizó información sobre la educación y la figura del maestro, a partir de las opiniones y observaciones de los participantes. Se generaron ideas y propuestas conceptuales y se compartieron y discutieron experiencias. En la última sesión se produjo el «filtrado de las ideas» que debían de estar presentes en el diseño del cartel.

La finalidad del taller fue que los jóvenes identificaran problemas, expresaran sus opiniones, analizaran la realidad, propusieran alternativas, razonaran, tomaran decisiones y negociaran con sus compañeros para consensuar una única propuesta. Se trató, en definitiva, de que participaran en un proceso colectivo de creación encaminado a generar vínculos y sentimiento de pertenencia al grupo. El propósito fue, además, el de contribuir a mejorar su capacidad de identificación y resolución de problemas.

Por lo que se refiere a la jornada de trabajo con la diseñadora gráfica, se presentaron y discutieron las ideas surgidas en las tres sesiones anteriores para determinar su viabilidad. De ahí nacieron los primeros bocetos del cartel y los jóvenes decidieron la opción final. Con ello, no solo participaron en un proceso de co-creación sino que mostraron su capacidad de argumentación y de negociación.

Finalmente, en el marco de la Escuela de Verano, dos de los participantes, en representación de sus compañeros, hablaron en público de su experiencia. Con esta presentación, se perseguía incrementar su autoestima, haciéndoles conscientes de que su trabajo había tenido sentido y podía ser útil para otras personas.

\section{Conclusiones}

En este proyecto, el diseño se ha empleado como medio para ejercer una intervención socioeducativa ${ }^{10}$. Las actividades no se concibieron con un sentido finalista sino como un medio para tratar otros asuntos

10 Siguiendo a Melendro et al. (2014: s.p.), entendemos por intervención «socioeducativa» una «acción social de naturaleza y alcance educativo» cuya finalidad es, según Caride, dar «respuesta a un complejo entramado de necesidades sociales de los individuos, restableciendo y ampliando las oportunidades educativas de las personas y de los colectivos sociales en la vida cotidiana» (Ibídem). 
fundamentales en la vida de los jóvenes. En ese sentido, el proyecto quiso responder a la necesidad de los educadores de centrar el trabajo en acciones concretas que promuevan la motivación.

Encuanto a la definición de objetivos, se estableció como general el de activar las capacidades, potencialidades, habilidades y conocimientos de los jóvenes que participaban en el proyecto.

El taller de co-diseño sirvió también para que los jóvenes debatieran y reflexionaran sobre la enseñanza que habían recibido o estaban recibiendo y sobre cómo desearían que fuera. Como dijo uno de los participantes: «me gustaría que la escuela fuera la cooperativa de los idealistas». La Asociación Rosa Sensat estuvo al corriente del proceso, puesto que dos personas de su equipo asistieron a la mayoría de las sesiones.

Si bien se había planteado una evaluación del proyecto flexible, formativa y procesual, finalmente, solo pudo llevarse a cabo la parte relacionada con el diseño, debido a una serie de cambios en la estructura del equipo de educadores del Casal que impidieron hacerla de manera global.

Sin embargo, la reunión de valoración realizada con los diseñadores de Designit permitió concluir que algunas herramientas no habían funcionado de manera eficiente (el uso de las piezas de Lego, por ejemplo, demostró sus limitaciones cuando se trata de que adolescentes y jóvenes se enfrenten a conceptos abstractos) pero, también, que el proceso había servido para detectar problemas personales (exceso de timidez, conflictos familiares, carencias materiales, inquietudes sobre el futuro, etc..., que los jóvenes no habían manifestado en sus entrevistas con los educadores) y, también, para identificar habilidades (lingüísticas, de liderazgo, de razonamiento lógico, etc...) que bien impulsadas pueden constituir la base de un asesoramiento personal y profesional adecuado, por parte de los educadores. Fue útil, también, para que algunos jóvenes recién llegados al centro (y a la ciudad) se integraran con más facilidad pues establecieron nuevos vínculos sociales. Para los participantes constituyó una experiencia estimulante y divertida, como comentaron en el diálogo posterior con ellos.

Finalmente, me gustaría comentar que un aspecto destacable fue la colaboración entre diversas personas y organizaciones. Así, la concepción, elaboración y coordinación del proyecto de intervención social corrió a mi cargo. Fue aprobado por los educadores sociales del Casal (Sara Ruiz — coordinadora del centro- y Josep Vives) quienes, además, supervisaron a los jóvenes durante el proceso; los diseñadores Aurélie Glorieux y Martin Dubuisson, con la ayuda de Alessandra Luis, miembros de la empresa internacional de diseño Designit ${ }^{11}$, se ocuparon del taller de co-diseño, previamente planificado con Mercè Graell (en 2017, Directora de Diseño estratégico de Designit). Asimismo, la diseñadora gráfica Pilar Villuendas participó en la jornada de co-creación del cartel y llevó a cabo el diseño de este, con la colaboración de Alicia Gómez, también diseñadora gráfica.

11 Designit colaboró cediendo el trabajo de sus diseñadores. Los jóvenes participantes recibieron una «recompensa» por su participación, consistente en una visita a Port Aventura. Estudios recientes han puesto de relieve que el cerebro adolescente es más sensible a la recompensa, algo que les posibilita aprender mejor de sus experiencias y de su entorno. ADG-FAD se encargó de conseguir el patrocinio para la «recompensa», gracias a las gestiones de su equipo de Secretaría. 


\section{BIBLIOGRAFÍA}

DEL VAL, T. (2011). Creatividad en el Teatro Infantil de Engrata. Estudio de la Compañía. Trabajo Final de Máster. Ponte de Lima/Porto: Universidade Fernando Pessoa.

LOSADA, S. (2016). Metodología de la intervención social, Madrid: Síntesis.

MARGOLIN, V.Y MARGOLIN, S.(2002). «Social Model» of Design: Issues of Practice and Research, Design Issues, vol. 18 (4), pp. 24-30.

MARTÍNEZ-CELORRIO, X. (2015). Potenciación socioeducativa de niños y jóvenes en un Raval intercultural. Barcelona: Universitat de Barcelona. Recuperado de http://diposit.ub.edu/dspace/bitstream/2445/99425/6/ INFORME\%20Potenciaci\%C3\%B3n\%20socioeducativa\%20de\%20ni\%C3\%B1os\%20y\%20 j\%C3\%B3venes\%20en\%20un\%20Raval\%20intercultural.pdf.

MELENDRO, M. (dir.) (2009). Estrategias educativas con adolescentes y jóvenes en dificultad social. El tránsito a la vida adulta en una sociedad sostenible. Madrid: UNED.

MELENDRO ESTEFANÍA, M. (coord.) (2014). Estrategias eficaces de intervención socioeducativa con adolescentes, Madrid: UNED. (Versión Kindle).

RICART, M.Y SAURÍ, E. (2009). Processos creatius transformadors. Els projectes arístics d'intervenció comunitària protagonitzats per joves a Catalunya. Barcelona: Ediciones del Serbal.

RUIZ, S. (2016). Document de Formulació de Projecte. Casal Jove Atles. Barcelona: Casal dels Infants. (Documento de circulación interna).

SANDERS, E.B.Y STAPPERS, P. J. (2008). Co-creation and the new landscapes of design. Recuperado de (http://studiolab.ide.tudelft.nl/manila/gems/contextmapping/PreprintDraft.pdf).

SEGADO SÁNCHEZ-CABEZUDO, S. (2012). Nuevas tendencias en Trabajo Social con familias. Una propuesta para la práctica desde el empowerment. Madrid: Trotta.

VON BERTANLANFFY, L. (1976). Teoría general de los sistemas. Fundamentos, desarrollo y aplicaciones. México: Fondo de Cultura Económica. 